\title{
MHD OSCILLATIONS OBSERVED IN THE SOLAR PHOTOSPHERE WITH THE MICHELSON DOPPLER IMAGER
}

\author{
A.A. NORTON AND R.K. ULRICH \\ Dept. of Phys. and Astron., Univ. of California at Los Angeles
}

AND

\author{
R.S. BOGART, R.I. BUSH AND J.T. HOEKSEMA \\ Hansen Experimental Phys. Laboratory, Stanford Univ.
}

Magnetohydrodynamic (MHD) oscillations are observed in the solar photosphere with the Michelson Doppler Imager (MDI). The absorption of acoustic waves by sunspots (Braun et al., 1988) and the possible conversion of acoustic waves into MHD waves (Spruit et al., 1992) provide a plausible basis for the existence of MHD waves in the solar atmosphere and motivate this attempt at observation.

Images of surface velocity, V, and magnetic flux, B, with 4" spatial and 60 second temporal resolution are analyzed. A 2-D gaussian aperture with 10" FWHM is applied to the data in regions of sunspot and plage and the averaged signal is returned each minute. Sunspot passage is followed using a modified Carrington rate. Representative signals are shown in Figure 1. Significant power is observed in the B oscillations with 5 minute periods, as seen in Figure 2. It is assumed that the large amplitude acoustic waves with 5 minute periods are the driving mechanism behind the $\mathrm{B}$ oscillations.

To determine if the B oscillations are instrumental or solar in origin, the effect of misregistration between left and right circularly polarized (LCP, RCP) images is investigated and found not to be the cause of the observed B oscillations, see Figure 3 . If the B signal were due to misregistration, the sunspot signal would decrease to the level of noise at a given misregistration, as found in quiet sun.

Ulrich (1996) proposed that phase relations between B and V contain the signatures of Alfénic and/or magnetoacoustic waves, which have 0 and \pm 60 second time lags, respectively. Phase angles between V and B oscillations are calculated for 19 sunspot and plage regions, see Figure 4. An average weighted time lag of -67 seconds is consistent with magnetoacoustic waves.

\section{References}

Braun DC, LaBonte BG, Duvall TL: 1988, 'The absorption of high-degree p-mode oscillations in and around sunspots,' ApJ. 335, 1015

Spruit HC, Bogdan TJ: 1992, 'The conversion of p-modes to slow modes and the absorption of acoustic waves by sunspots,' $A p J$. 391L, 109

Ulrich, RK: 1996, 'Observations of magnetohydrodynamic oscillations in the solar atmosphere with properties of Alfvén waves,' $A p J .465,436$ 

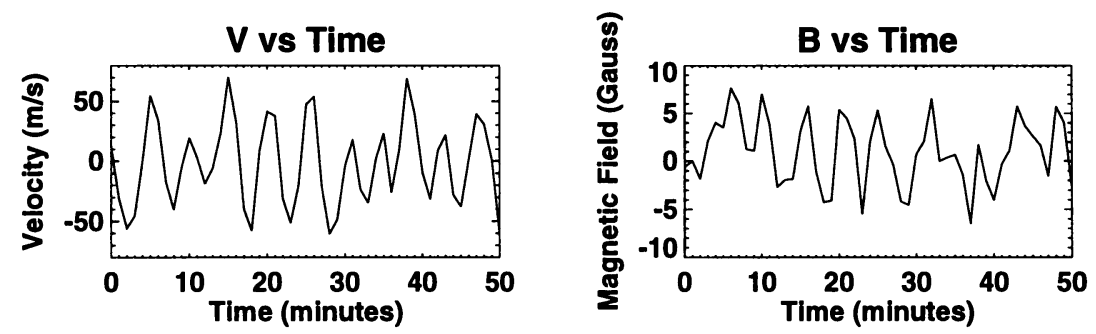

Figure 1. Representative V and B signals from an Aug 30, 1996 sunspot region. Signals are detrended with a 30 minute boxcar average. Average $V$ and $B$ values are subtracted.
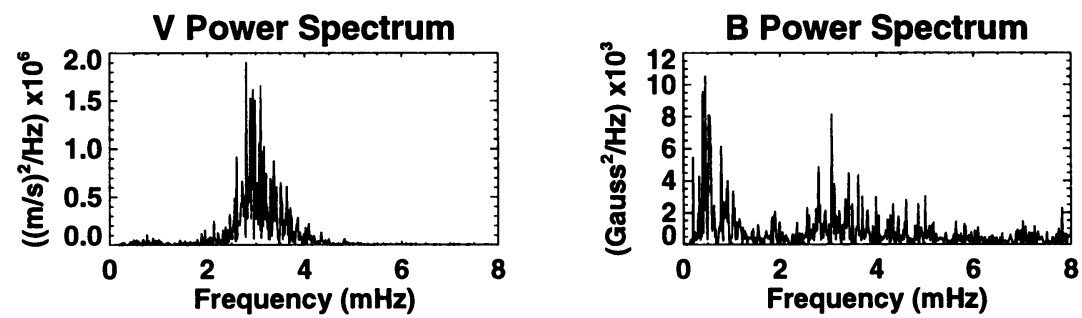

Figure 2. Velocity and magnetic power spectra from an August 30, 1996 sunspot region.
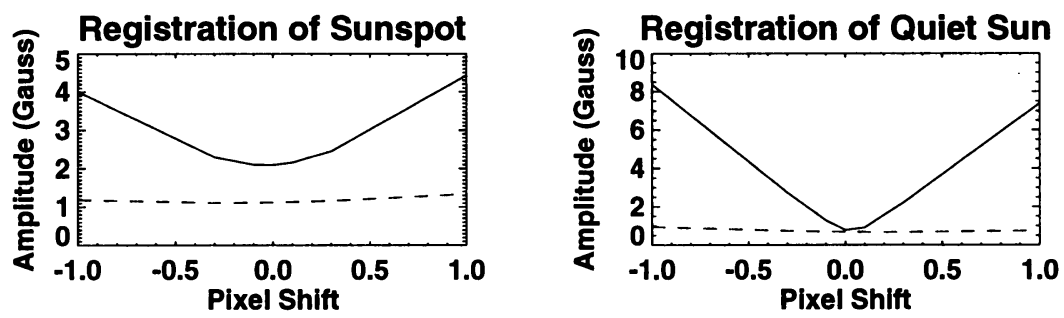

Figure 3. LCP and RCP images are shifted against each other to generate misregistered datasets. Resulting B signal (solid) and noise (dashed) amplitude are plotted vs N-S misregistration for sunspot (left) and quiet sun (right).

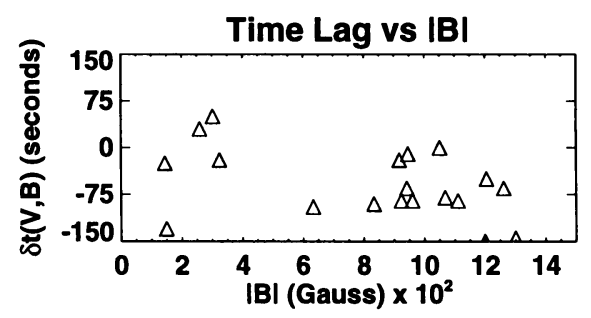

Figure 4. The time lag between $\mathrm{V}$ and $\mathrm{B}$ is plotted vs absolute magnetic field strength. V is cross correlated with $\mathrm{B}$ and the phase is calculated as the time lag, $\delta$, corresponding to the largest cross correlation coefficient within a 300 second period. 